\title{
Isolation, DNase-cross-Coagulase test and antimi- crobial resistance test on Staphylococcus along beef abattoir line in Addis Ababa Ethiopia
}

\author{
Adem Hiko* \\ College of Veterinary Medicine, Haramaya University, Po. Box. 138, Dire Dawa, Ethiopia. \\ Correspondence: E. mail: adex.2010ph@gmail.com
}

\begin{abstract}
Staphylococci are responsible for foodborne infection and intoxication with the spread of antibiotic resistance. The aims of the study were to investigate beef abattoir line contamination with Staphylococcal, to evaluate DNase test for alternative of the tube coagulase test, and to assess isolates drug resistance in Ethiopia. A total of 169 samples from slaughter environment, raw beef at inspection and at public supply along Addis Ababa Abattoir Enterprise line were examined for Staphylococci. The isolates were tested against DNase, tube plasma coagulase, and eight medicinal drugs. A total proportion of $35.5 \%$ (60/169) isolates with $13.6 \%$ S. aureus and $21.9 \%$ coagulase negative Staphylococcus (CNS) were observed. All sampling locations were found positive for Staphylococcus environmental samples ranged from $18.2 \%-46.2 \%$ with no difference ( $p>0.05)$ among locations. In raw beef, it was $23.5 \%$ at abattoir and $52.9 \%$ at butchers. Three (1.9\%), $13(7.7 \%)$ and 23 (13.6\%) of locations were positive for S. aureus only, CNS only and both as a mixed, respectively. Of all 60 Staphylococci isolates, the DNase test and coagulase tests were in agreement for 56 isolates ( 21 for positive, 35 for negative) showing DNase test was strong agreement with the gold standard test (coagulase tests), kappa=0.86)). S. aureus was $38.3 \%$ but CNS was $61.7 \%$. Resistant isolates were observed for trimethoprim (35.0\%), polymyxin-B (33.7\%), oxytetracycline (31.7\%), trimethoprim-sulfamethoxazole (20.0\%), chloramphenicol (8.3\%), oxacillin (6.7\%), and gentamycin $(5.0 \%)$ but not for tetracycline. Thirty-one $(51.7 \%)$ isolates were resistant for at least one drug with multiple drugs resistance (MDR) of three to six in 17 isolates. Contamination of all sampling locations with Staphylococcus including with resistant isolate to medically used drugs warrants the application of good hygienic practices along the abattoir line. Due to availability and cost effectiveness, DNase can be used as alternative to the gold standard, coagulase test, for diagnosis of Staphylococcus.
\end{abstract}


Key words: Abattoir line; beef; drug resistance; foodborne intoxication; Staphylococci

\section{Introduction}

Staphylococcus species are classified as classical pathogen, causing infections (Waldvogel, 1995; Lowry, 1998). But, heterogeneous group of coagulase-negative Staphylococci (CNS) those historically as being less or nonpathogenic (Becker et al., 2014). They are differentiated based on their diagnostic reaction. Carriers are at higher risk of infection and they are presumed to be an important source of spread of $S$. aureus strains among individuals, to food and food production and processing chain (Muto et al., 2003; Kazakova et al., 2005; Miller and Diep, 2008). A fundamental biological property of $S$. aureus is the ability to asymptomatically colonize normal people. Approximately $30 \%$ of humans are asymptomatic nasal carriers of S. aureus (Kluytmans et al., 1997; Gorwitz et al., 2008). Due to patient and procedure related changes, CNS also represent one of typical opportunistic major nosocomial pathogens having a substantial impact on human life and health, and also food associated saprophytes resulted in clinically manifested infections (Becker et al., 2014).

The ideal identification of clinical $S$. aureus isolates requires a battery of tests and this is costly in resource limited settings. S. aureus identification was routinely done using either human or sheep plasma. Study was also conducted using mannitol salt agar and the deoxyribonuclease (DNase) test for improving the efficiency of the tube coagulase test in resource limited settings (Kateete et al., 2010). Indicating the absence of single phenotypic test (including tube coagulase) that can guarantee as reliable method for identification of $S$. aureus, Kateete et al. (2010) suggested the need for sequel testing of the isolates with Mannitol salt agar, DNase and Tube coagulase. On the other hand, the coagulase test is a golden standard method to differentiate $S$. aureus from coagulase negative Staphylococcus (Koneman et al., 1997). Coagulase production can be detected using either slide coagulase test or the tube coagulase test. The tube coagulase test is better than the slide coagulase test in that it detects the secreted extracellular free coagulase which reacts with a substance in plasma called "Coagulase-Reacting Factor" (CRF) to form a complex clot (Koneman et al., 1997; Winn et al., 2006). 
The extended use and misuse of antibiotics in agriculture, livestock farming and human medicine have rapidly facilitated the emerging of resistant bacteria strains including Staphylococci (Valsangiacomo et al., 2000). Antibiotic resistance leads to prolonged hospital stay and increased costs in terms of treatment (Kitara et al., 2011). Multidrug resistance is norm among Staphylococci pathogens (Lowy, 2003).

Study along milk production, Gizaw (2014) reported Staphylococci species at $47.1 \%$ from udder milk, $58 \%$ from tank milk of the selected dairy farms, $34.4 \%$ from cow bucket swab, 38\% from farm tank swab, 38\% from milkers hand and $70 \%$ from nasal swab of milkers. Similarly, prevalence of $38.5 \%$ from carcass swab, 37.8\% from knife swab, 48.6\% from slaughter line swab, $37.8 \%$ from abattoir worker hand and $46.7 \%$ nasal swab of butchers were reported in Ethiopia (Gizaw, 2014). Indicating the significance of drug resistance, pooled prevalence of methicillin resistant $S$. aureus was reported at rate of $32.5 \%$ from several report meta-analysis data in Ethiopia (Eshetie et al., 2016). On the other hand, structured abattoir line-based Staphylococci survey with DNase test were not yet conducted in Ethiopia. Therefore, the aims of the study were to investigate beef abattoir line contamination with Staphylococcal strain, to evaluate DNase test as alternative of the tube coagulase test and to assess drug resistance status of the isolates against medicinal drugs frequently used in Ethiopia.

\section{Materials and Methodology}

\section{Abattoir lines description}

The study was conducted at Addis Ababa Abattoir Enterprise (AAAE) located in Addis Ababa city. Multi-purpose cattle stocks purchased from extensive or semi-intensive management systems in different parts of the Ethiopia, transported to AAAE and slaughtered for human consumption were used. The AAAE has a capacity to slaughter up to 1,200 cattle in 8 hours with a staff of about 700 persons. After slaughter, carcasses were examined through routine meat inspection procedures. The raw beef, immediately or after a short cooling interval were loaded into meat transport trucks and delivered to city butcheries. Butcheries are mostly small open-stall shops, handling the meat at $20^{\circ} \mathrm{C}-$ $27^{\circ} \mathrm{C}$, which is the ambient temperature in Addis Ababa City. 


\section{Sampling and sample types}

Samples were taken from December 2011 to April 2012 from the abattoir and beef retailer butchery. In the abattoir, different samples were taken from the operation environment. Sample of raw beef were also taken directly after quality inspection at abattoir. Following the beef identification number, same raw beef product were sampled at butchery houses located at Addis Ababa. A total of 169 samples from 10 sampling locations (Table 1) were taken. For swabs, a $50 \mathrm{~cm}^{2}$ area was swabbed with sterilized gauze moistened with normal saline solution. Water $(20 \mathrm{~mL})$ sample was filled directly from the tap into sterile calibrated glass bottles. Meat samples (34 samples from AAAE and 34 samples from butcheries) were taken aseptically and placed in sterile stomacher bags. The number and types of samples were described within Table 1 with the results. Samples were immediately transported to Microbiology Laboratory, Akililu Lemma Institute of Pathobiology, Addis Ababa University (ALIPB$\mathrm{AAU})$, Ethiopia on the day of sampling using an ice box at $+4^{\circ} \mathrm{C}$.

\section{Sample preparation}

Each sample was aseptically taken. For pre-enrichment, buffered peptone water (Merck, Darmstadt, Germany) was used and the inoculate were incubated at $37^{\circ} \mathrm{C}$ for $18-20$ hours. The first 1:10 dilution was homogenized with a Stomacher 400 (Seward Laboratory, London, UK) and incubated at $37^{\circ} \mathrm{C}$ for $18-20$ hours to be used as pre-enrichment (Monttville et al., 2012; USDA, 2012).

\section{Staphylococcus isolation and characterization}

A loopfull of pre-enrichment was inoculated on whole-hen-egg based Kranep Agar (Merck, Germany). One to five presumptive Staphylococci colonies were taken per-each sample. Colonies were screened using $3 \%$ hydrogen peroxide. The presumptive positive $S$. aureus colonies isolate was transferred to Standard-II Nutrient Agar (Merck, Germany) incubated at $37^{\circ} \mathrm{C}$ for $24 \mathrm{hr}$, stored at $+4^{\circ} \mathrm{C}$. The strain was exposed to deoxyribonuclease (DNase) and tube plasma coagulase test in parallel. An overnight cultured colony was inoculated in forms of line (Fig. 1) on DNase Agar (Merck, Germany) and incubated for 18-24 $\mathrm{hr}$ at $37^{\circ} \mathrm{C}$. The culture was over flushed with $1 \mathrm{ml} 1 \mathrm{~mole} / \mathrm{ml}$ Hydrochloric acid. Based up on DNA digestion zone of clear transparence surrounding the culture, the strains were termed as DNase positive. Parallel to DNase test, tube plasma coagulase test (Bactident Merck, Germany) was done. The colony was inoculated in Brain Heart Infusion (BHI) (Merck, Germany) incubated at $37^{\circ} \mathrm{C}$ 
for $18-24 \mathrm{hr}$. A $0.5 \mathrm{ml}$ of rabbit plasma (Bactident Merck, Germany) was added in to sterile test tube followed by addition of $0.1 \mathrm{ml}$ of culture suspension. The mixture was incubated in $37^{\circ} \mathrm{C}$ water bath with $1 \mathrm{hr}$ interval up to $4 \mathrm{hr}$ with registration of degrees of coagulation. Finally, the tubes were kept overnight at room temperature and re-examined.

\section{Considered and used test protocol}

In Staphylococcus identification, coagulase production can be detected using either the slide coagulase test or the tube coagulase test (Winn et al., 2006). The tube coagulase is better than the slide coagulase test in that it detects the secreted extracellular free coagulase which reacts with a substance in plasma called "Coagulase-Reacting Factor" to form a complex clot (Koneman et al., 1997; Winn et al., 2006). Thus, coagulase testing was used as gold standard for differentia between $S$. aureus and coagulase negative Staphylococcus (CNS). Control reactions included templates of Staphylococcus aureus ATCC 25923 (positive control), Staphylococcus epidermidis ATCC 12228 and nuclease free water (negative controls).

\section{Ethical Considerations}

Samples were also collected from adult personnel's hands who are working in the abattoir. Individuals involved on the sample were informed regarding the details of the research objectives. Hence, informed consent was collected.

\section{Antimicrobial resistance test}

The test was done on Mueller-Hinton agar (Oxoid, Hampshire, England) using Kirby Bauer disc diffusion criteria of the National Committee for Clinical Laboratory Standards (Bauer et al., 1996; CLSI, 2012). The following antimicrobial substances (Oxoid UK) were used: chloramphenicol (C $50 \mu \mathrm{g})$, gentamycin (CN $10 \mu \mathrm{g}$ ), oxytetracycline (OT $30 \mu \mathrm{g}$ ), oxacillin (OX $1 \mu \mathrm{g})$, polymyxin B (PB 300U), tetracycline (TE $10 \mu \mathrm{g}$ ), trimethoprim-sulfamethoxazole (STX 1.25/23.75 $\mu \mathrm{g}$ ) and trimethoprim (W $5 \mu \mathrm{g}$ (Oxoid, Hampshire, England). They were selected using the fact that they are used in health sectors of Ethiopia and their availability on the markets. The isolates were sub-cultured on Nutrient agar (Merck, Germany) and incubated at $37^{\circ} \mathrm{C}$ for $24 \mathrm{hrs}$. They were then inoculated into $5 \mathrm{ml}$ of Brain Heart Infusion broth (BHI) (Merck, Germany) and again incubated for $1 \mathrm{hr}$ at $37^{\circ} \mathrm{C}$. The inoculum density was standardized using a $0.5 \mathrm{McF}$ arland standard. Volume of $0.1 \mathrm{ml}$ of standardized culture was

Ethiop. Vet. J., 2019, 23 (1), 90-110 
spread on Mueller-Hinton agar (Oxoid, Hampshire, England). The antibiotics disc was mounted and incubated at $37^{\circ} \mathrm{C}$ for $18-20$ hours. Based on the diameter of inhibition zone, the results were recorded as susceptible, intermediate and resistant according to the rules of National Committee for Clinical Institute laboratory standards (CLSI, 2012).

\section{Data analysis}

Data were entered in to Microsoft Excel 2007® (Microsoft Corp., Redmond, USA) and analyzed using Excel, State 11, and SPSS version 20 (IBM Corp., Armonk, USA). Percentage and 95\% confidence intervals (CI) of the percentage were used to demonstrate prevalence differences between and among the sampling occasions and types of samples. Using the coagulase test as gold standard (Winn et al., 2006; Koneman et al., 1997), the diagnostic test agreement (Kappa), sensitivity and specificity were also calculated. The cut-off Kappa value and interpretation was according to Viera, and Garrett (2005).

\section{Results}

Out of the total 169 samples, $35.5 \%$; $95 \%$ CI $28.6 \%$-42.9\% were found positive for Staphylococcus. The prevalence ranges of $18.2 \%$ in hooks to $46.2 \%$ from personal hand swab with no difference $(p>0.05)$ were observed in environmental samples. In raw beef, it was $23.5 \%$ from when ready for distribution at abattoir and $52.9 \%$ in post distribution at butchers (Table 1 ). 
Table 1: Staphylococcus by sampling locations and sample types along the Addis Ababa Abattoir Enterprise beef abattoir line.

\begin{tabular}{|c|c|c|c|c|c|c|}
\hline \multirow{2}{*}{\multicolumn{2}{|c|}{$\begin{array}{l}\text { Origin of } \\
\text { sample }\end{array}$}} & \multirow{2}{*}{$\begin{array}{l}\text { Processing } \\
\text { stages/position }\end{array}$} & \multirow[b]{2}{*}{$\begin{array}{l}\text { Sampling } \\
\text { location }\end{array}$} & \multirow{2}{*}{$\begin{array}{c}\text { Total } \\
\text { № of } \\
\text { sample }\end{array}$} & \multicolumn{2}{|c|}{ Total Staphylococcus } \\
\hline & & & & & № (\%) & $\begin{array}{c}95 \% \mathrm{CI} \\
\text { percentage. }\end{array}$ \\
\hline \multirow{8}{*}{\multicolumn{2}{|c|}{ 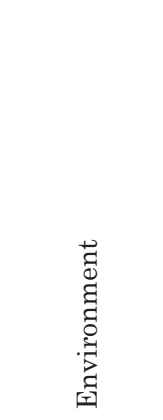 }} & \multirow{4}{*}{$\begin{array}{l}\text { Before beginning } \\
\text { of operation }\end{array}$} & Personnel's hands & 13 & $6(46.2)$ & $23.0-71.1$ \\
\hline & & & Aprons & 14 & $6(42.9)$ & $21.3-67.7$ \\
\hline & & & Knives & 13 & $4(30.8)$ & $12.8-58.1$ \\
\hline & & & Hooks & 11 & $2(18.2)$ & $5.5 .-48.4$ \\
\hline & & Cleaning water & Tap water & 12 & $3(25.0)$ & $9.1-53.8$ \\
\hline & & At carcass splitting & Rooms & 17 & $5(29.4)$ & $13.3-53.5$ \\
\hline & & Refrigeration & Refrigerators & 10 & $3(30.0)$ & $10.9-60.9$ \\
\hline & & Meat transport & $\begin{array}{l}\text { Meat transport } \\
\text { trucks }\end{array}$ & 11 & $5(45.5)$ & $21.1-72.3$ \\
\hline \multirow{2}{*}{\multicolumn{2}{|c|}{ 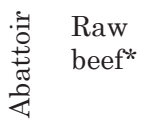 }} & $\begin{array}{l}\text { After washing for } \\
\text { distribution }\end{array}$ & Beef inspection & 34 & $8(23.5)$ & $12.5-40.1$ \\
\hline & & Sub total & & 135 & $42(31.1)$ & 23.4-39.6 \\
\hline \multicolumn{2}{|c|}{ Butchers } & $\begin{array}{l}\text { Butchers, } 6-8 \\
\text { hours post delivery }\end{array}$ & $\begin{array}{l}\text { Beef for } \\
\text { consumption }\end{array}$ & 34 & $18(52.9)$ & $35.1-70.2$ \\
\hline \multicolumn{2}{|c|}{ Total } & & & 169 & $60(35.5)$ & $28.6-42.9$ \\
\hline
\end{tabular}

From a total sample, $13.6 \%$ S. aureus and $21.9 \%$ CNS were observed (Table 2). Among environmental samples, S. aureus ranged from $0 \%$ on knives to $36.4 \%$ in meat transport trucks while CNS ranged from $0 \%$ on hooks to $46.2 \%$ on personnel's hands swabs. In raw beef, S. aureus was $8.8 \%$ at ready for distribution but raised to $20.6 \%$ post-delivery at public supply. Similarly, CNS was $14.7 \%$ at ready for distribution but raised to $32.4 \%$ post-delivery at public supply. 
Table 2: Distribution of S. aureus and CNS by sampling locations and sample types along Addis Ababa Abattoir Enterprise beef abattoir line.

\begin{tabular}{|c|c|c|c|c|c|}
\hline \multicolumn{2}{|c|}{ Origin of sample } & Sampling location & $\begin{array}{c}\text { TNo of } \\
\text { sample }\end{array}$ & $\begin{array}{c}\text { S. aureus № } \\
(\mathbf{\%})\end{array}$ & CNS № (\%) \\
\hline \multirow{10}{*}{ 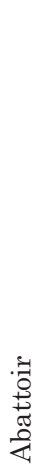 } & \multirow{8}{*}{  } & Personnel's hands & 13 & $3(23.1)$ & $3(23.1)$ \\
\hline & & Aprons & 14 & $2(14.3)$ & $4(28.6)$ \\
\hline & & Knives & 13 & 0 & $4(30.8)$ \\
\hline & & Hooks & 11 & $2(18.2)$ & 0 \\
\hline & & Tap water & 12 & $1(8.3)$ & $2(16.7)$ \\
\hline & & Rooms & 17 & $2(11.8)$ & $3(17.6)$ \\
\hline & & Refrigerators & 10 & $2(20.0)$ & $1(10.0)$ \\
\hline & & Meat transport trucks & 11 & $1(9.1)$ & $4(36.4)$ \\
\hline & Raw beef* & Beef inspection & 34 & $3(8.8)$ & $5(14.7)$ \\
\hline & Subtotal & & 135 & $16(11.9)$ & $26(19.3)$ \\
\hline \multicolumn{2}{|c|}{ Butchers } & Beef for consumption & 34 & $7(20.6)$ & $11(32.4)$ \\
\hline \multicolumn{2}{|c|}{ Total } & & 169 & $23(13.6)$ & $37(21.9)$ \\
\hline
\end{tabular}

Mixed occurrence of $S$. aureus and CNS by sampling locations was shown in Table 3. S. aureus only, CNS only and co-occurrence were $1.9 \%, 7.7 \%$ and $13.6 \%$ respectively. Except on knives and hooks, mixed occurrences of S. aureus and CNS were observed in all other sampling locations. 
Table 3: Mixed occurrence of $S$. aureus and CNS by sampling locations and sample types along Addis Ababa Abattoir Enterprise beef abattoir line.

\begin{tabular}{|c|c|c|c|c|c|c|}
\hline \multicolumn{2}{|c|}{ Origin of sample } & Sampling location & $\begin{array}{l}\text { Total № of } \\
\text { sample }\end{array}$ & $\begin{array}{c}\text { S. } \\
\text { aureus } \\
\text { only № } \\
(\%)\end{array}$ & $\begin{array}{c}\text { CNS } \\
\text { only № } \\
(\%)\end{array}$ & $\begin{array}{c}\text { Mixed } \\
\text { isolation } \\
\text { № (\%) }\end{array}$ \\
\hline \multirow{10}{*}{ 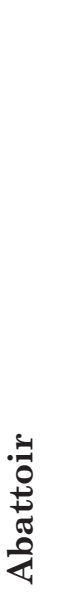 } & & Personnel's hands & 13 & 0 & 0 & $3(23.1)$ \\
\hline & & Aprons & 14 & 0 & $2(14.3)$ & $2(14.3)$ \\
\hline & & Knives & 13 & 0 & $4(30.8)$ & 0 \\
\hline & & Hooks & 11 & $\begin{array}{c}2 \\
(18.2)\end{array}$ & 0 & 0 \\
\hline & \multirow{4}{*}{ 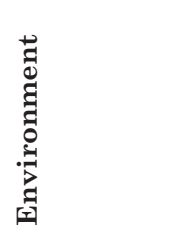 } & Tap water & 12 & 0 & $1(8.3)$ & $1(8.3)$ \\
\hline & & Rooms & 17 & 0 & $1(5.9)$ & $2(11.8)$ \\
\hline & & Refrigerators & 10 & $1(10.0)$ & 0 & $1(10.0)$ \\
\hline & & $\begin{array}{l}\text { Meat transport } \\
\text { trucks }\end{array}$ & 11 & 0 & $1(9.1)$ & $4(36.4)$ \\
\hline & Raw beef* & Beef inspection & 34 & 0 & $2(5.9)$ & $3(8.8)$ \\
\hline & Subtotal & & 135 & $3(2.2)$ & $9(6.6)$ & $16(11.9)$ \\
\hline \multicolumn{2}{|c|}{ Butchers } & Beef for consumption & 34 & 0 & $4(11.8)$ & $7(20.6)$ \\
\hline \multicolumn{2}{|c|}{ Total } & & 169 & $3(1.9)$ & $13(7.7)$ & $23(13.6)$ \\
\hline
\end{tabular}

* = Raw beef at abattoir

The coagulase test was used as gold standard. Of all 60 isolates exposed to DNase (Figure 1) and coagulase tests, 21 were positive for both tests whereas 35 were negative for both test reactions showing (Kappa $=0.86$; 95\% CI $=0.73$ 0.99) Sensitivity $=91.3 \%$ and Specificity $=94.6 \%)($ Table 4$)$. Out of the total 60 isolates, 23 (38.3\%) and 37 (61.7\%) isolates were coagulase positive Staphylococcus (CPS) and coagulase negative Staphylococcus (CNS), respectively. 


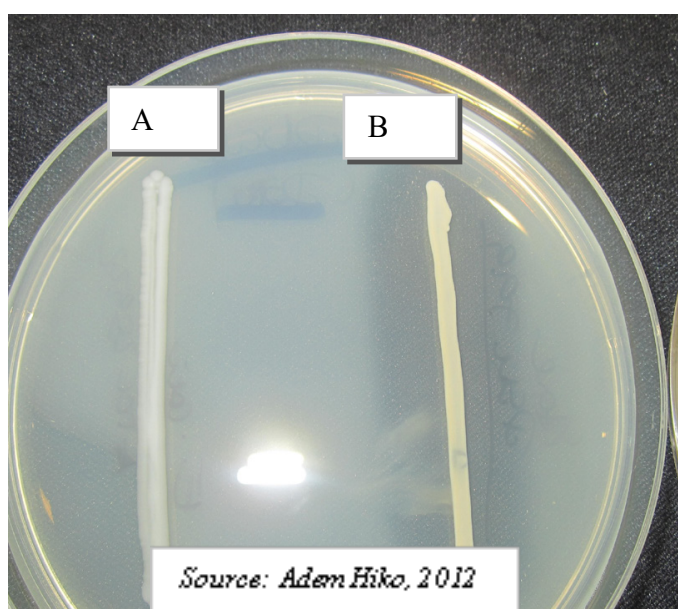

Figure 1: DNase test reaction of isolated Staphylococcus: A) DNase negative, B) DNase positive (Clear zone of DNA digestion).

Table 4: DNase-cross-Coagulase test reactions of Staphylococcal isolates from the Addis Ababa Abattoir Enterprise beef abattoir line

\begin{tabular}{|c|c|c|c|c|}
\hline \multicolumn{2}{|c|}{ Diagnostic test } & \multicolumn{2}{|c|}{ № of tube coagulase } & \multirow[t]{2}{*}{ Total № (\%) } \\
\hline & & Positive & Negative & \\
\hline \multirow[t]{2}{*}{ № of DNase } & Positive & $21^{*}$ & $2^{* *}$ & $23(38.3)$ \\
\hline & Negative & $2^{* * * *}$ & $35^{* * * *}$ & $37(61.7)$ \\
\hline Total № (\%) & & $23(38.3)$ & 37 (61.7) & $60(100)$ \\
\hline
\end{tabular}

\section{Antimicrobial Resistance test}

The 60 Staphylococcus isolates were subjected to 8 types of antibiotics for antimicrobial resistance test. Out of the total isolates, $35.0 \%$ were resistance to trimethoprim, $33.7 \%$ for polymyxin B, $31.7 \%$ for oxytetracycline, $20.0 \%$ for trimethoprim-sulfamethoxazole, $8.3 \%$ for chloramphenicol, $6.7 \%$ for oxacillin, $5.0 \%$ for gentamycin and no resistance was observed for tetracycline (Table 5). 
Table 5: Drug resistance profiles of the Staphylococcal isolates from the Addis Ababa Abattoir Enterprise beef abattoir line.

\begin{tabular}{|c|c|c|c|c|c|c|c|c|c|}
\hline \multirow{2}{*}{$\begin{array}{l}\text { Drugs } \\
\text { used }\end{array}$} & \multicolumn{3}{|c|}{ S. aureus $(n=23)$} & \multicolumn{3}{|c|}{ CNS (n = 37) } & \multicolumn{3}{|c|}{ Total $(\mathrm{N}=60)$} \\
\hline & S. № (\%) & $\begin{array}{l}\text { I. № } \\
\text { (\%) }\end{array}$ & $\begin{array}{l}\text { R. № } \\
\text { (\%) }\end{array}$ & $\begin{array}{l}\text { S. № } \\
\text { (\%) }\end{array}$ & $\begin{array}{l}\text { I. № } \\
\text { (\%) }\end{array}$ & $\begin{array}{l}\text { R. № } \\
\text { (\%) }\end{array}$ & $\begin{array}{l}\text { S. № } \\
(\%)\end{array}$ & $\begin{array}{l}\text { I. № } \\
\text { (\%) }\end{array}$ & $\begin{array}{l}\text { R. № } \\
\text { (\%) }\end{array}$ \\
\hline $\mathrm{TE}$ & $23(100)$ & 0 & 0 & $37(100)$ & 0 & 0 & $\begin{array}{c}60 \\
(100)\end{array}$ & 0 & 0 \\
\hline $\mathrm{CN}$ & $20(86.9)$ & $\begin{array}{c}1 \\
(4.3)\end{array}$ & $2(8.7)$ & $\begin{array}{c}36 \\
(97.3)\end{array}$ & 0 & $1(2.7)$ & $\begin{array}{c}56 \\
(93.3)\end{array}$ & $1(1.7)$ & $3(5.0)$ \\
\hline $\mathrm{OX}$ & $20(86.9)$ & 0 & $\begin{array}{c}3 \\
(13.1)\end{array}$ & $\begin{array}{c}32 \\
(86.5)\end{array}$ & $\begin{array}{c}4 \\
(10.8)\end{array}$ & $1(2.7)$ & $\begin{array}{c}52 \\
(86.6)\end{array}$ & $4(6.7)$ & $4(6.7)$ \\
\hline $\mathrm{C}$ & $19(80.6)$ & $\begin{array}{c}1 \\
(4.3)\end{array}$ & $\begin{array}{c}3 \\
(13.1)\end{array}$ & $\begin{array}{c}35 \\
(94.6)\end{array}$ & 0 & $2(5.4)$ & $\begin{array}{c}54 \\
(90.0)\end{array}$ & $1(1.7)$ & $5(8.3)$ \\
\hline $\mathrm{SXT}$ & 12() 52.2 & $\begin{array}{c}1 \\
(4.3)\end{array}$ & $\begin{array}{c}10 \\
(43.5)\end{array}$ & $\begin{array}{c}34 \\
(91.9)\end{array}$ & $1(2.7)$ & $2(5.4)$ & $\begin{array}{c}46 \\
(76.7)\end{array}$ & $2(3.3)$ & $\begin{array}{c}12 \\
(20.0)\end{array}$ \\
\hline OT & $10(43.5)$ & 0 & $\begin{array}{c}13 \\
(56.5)\end{array}$ & $\begin{array}{c}30 \\
(81.1)\end{array}$ & $1(2.7)$ & $\begin{array}{c}6 \\
(16.2)\end{array}$ & $\begin{array}{c}40 \\
(66.7)\end{array}$ & $1(1.7)$ & $\begin{array}{c}19 \\
(31.7)\end{array}$ \\
\hline $\mathrm{PB}$ & $6(26.1)$ & 0 & $\begin{array}{c}17 \\
(73.9)\end{array}$ & $\begin{array}{c}34 \\
(91.9)\end{array}$ & 0 & $3(8.1)$ & $\begin{array}{c}40 \\
(66.7)\end{array}$ & 0 & $\begin{array}{c}20 \\
(33.7)\end{array}$ \\
\hline W & $7(30.4)$ & $\begin{array}{c}2 \\
(8.7)\end{array}$ & $\begin{array}{c}14 \\
(60.9)\end{array}$ & $\begin{array}{c}26 \\
(70.3)\end{array}$ & $\begin{array}{c}4 \\
(10.8)\end{array}$ & $\begin{array}{c}7 \\
(18.9)\end{array}$ & $\begin{array}{c}33 \\
(55.0)\end{array}$ & $\begin{array}{c}6 \\
(10.0)\end{array}$ & $\begin{array}{c}21 \\
(35.0)\end{array}$ \\
\hline
\end{tabular}

\section{Antimicrobial resistance at different sampling sites}

Two (5.9\%) to $12(35.3 \%)$ resistant Staphylococcus isolates from abattoir environmental samples was observed against all drugs used in this study. Similarly, resistant isolates to drugs used in this study were distributed on beef at abattoir and butchery. Exceptions are oxacillin resistant isolates were not observed on beef from abattoir and butchery while gentamycin resistant isolates were not observed in beef at abattoir (Table 6). 
Table 6: Distribution of resistant Staphylococcus isolates by origin of sample at Addis Ababa Abattoir Enterprise beef abattoir line.

\begin{tabular}{|c|c|c|c|c|c|c|c|c|}
\hline \multirow[t]{2}{*}{ Origin of sample } & \multirow{2}{*}{$\begin{array}{c}\text { № } \\
\text { test }\end{array}$} & \multicolumn{7}{|c|}{ № (\%) resistant Staphylococcus isolates* } \\
\hline & & $\mathrm{CN}$ & OX & $\mathrm{C}$ & SXT & OT & PB & W \\
\hline Environment** & 34 & $\begin{array}{c}2 \\
(5.9)\end{array}$ & $\begin{array}{c}4 \\
(11.8)\end{array}$ & $\begin{array}{c}12 \\
(35.5)\end{array}$ & $7(20.6)$ & $\begin{array}{c}11 \\
(32.4)\end{array}$ & $\begin{array}{c}11 \\
(32.4)\end{array}$ & $\begin{array}{c}12 \\
(35.3)\end{array}$ \\
\hline Beef at abattoir & 8 & 0 & 0 & $\begin{array}{c}3 \\
(37.5)\end{array}$ & $3(37.5)$ & $\begin{array}{c}2 \\
(25.0)\end{array}$ & $\begin{array}{c}5 \\
(62.5)\end{array}$ & $\begin{array}{c}3 \\
(37.5)\end{array}$ \\
\hline Beef at butcher & 18 & $\begin{array}{c}1 \\
(5.6)\end{array}$ & 0 & $\begin{array}{c}6 \\
(33.3)\end{array}$ & $2(11.1)$ & $\begin{array}{c}6 \\
(33.3)\end{array}$ & $\begin{array}{c}4 \\
(22.2)\end{array}$ & $\begin{array}{c}3 \\
(37.5)\end{array}$ \\
\hline Total & 60 & $\begin{array}{c}3 \\
(5.0)\end{array}$ & $4(6.7)$ & $\begin{array}{c}21 \\
(35.0)\end{array}$ & $\begin{array}{c}12 \\
(20.0)\end{array}$ & $\begin{array}{c}19 \\
(31.7)\end{array}$ & $\begin{array}{c}20 \\
(33.3)\end{array}$ & $\begin{array}{c}21 \\
(35.0)\end{array}$ \\
\hline
\end{tabular}

Note: $\mathrm{C}=$ Chloramphenicol; $\mathrm{CN}=$ Gentamycin; $\mathrm{TE}=\mathrm{OT}=$ Oxytetracycline; $\mathrm{OX}=$ Oxacillin; $\mathrm{PB}=$ Polymyxin $\mathrm{B} ; \mathrm{SXT}=$ Trimethoprim-Sulfamethoxazole; $\mathrm{W}=$ Trimethoprim; * = Tetracycline resistant isolates were not observed; ** = Abattoir Environment*

Higher resistant $S$. aureus the CNS were observed among the used drugs (Table 7$)$. Two (15.4\%) to ten $(76.9 \%)$ resistant $S$. aureus isolates to chloramphenicol, gentamycin, oxytetracycline, oxacillin, polymyxin B, trimethoprimsulfamethoxazole and trimethoprim were observed at abattoir environment (Table 7). On the other hand, resistant Staphylococcus isolates to chloramphenicol, gentamycin and oxacillin was not observed on beef both at abattoir and butchery. However, regardless of the number, high percentages of resistant Staphylococcus isolates to oxytetracycline, polymyxin B, trimethoprimsulfamethoxazole and trimethoprim were observed on beef at butchery than at abattoir. With regards to CNS, variable types of resistant isolates to the used drugs were observed at each sample origin. 
Table 7: Distribution of resistant Staphylococcus strain isolates by origin of sample at Addis Ababa Abattoir Enterprise beef abattoir line.

\begin{tabular}{|c|c|c|c|c|c|c|c|c|c|}
\hline \multirow{2}{*}{$\begin{array}{l}\text { Staphylo- } \\
\text { coccus } \\
\text { strain }\end{array}$} & \multirow{2}{*}{$\begin{array}{l}\text { Origin of } \\
\text { sample }\end{array}$} & \multirow{2}{*}{$\begin{array}{l}\text { № } \\
\text { test }\end{array}$} & \multicolumn{7}{|c|}{ № (\%) resistant Staphylococcus isolates* } \\
\hline & & & $\mathrm{CN}$ & OX & $\mathbf{C}$ & SXT & OT & PB & $\mathbf{W}$ \\
\hline \multirow[t]{4}{*}{ S. aureus } & Environment** & 13 & $\begin{array}{c}2 \\
(15.4)\end{array}$ & $\begin{array}{c}3 \\
(23.1)\end{array}$ & $\begin{array}{c}3 \\
(23.1)\end{array}$ & $\begin{array}{c}7 \\
(53.9)\end{array}$ & $\begin{array}{c}8 \\
(61.5)\end{array}$ & $\begin{array}{c}10 \\
(76.9)\end{array}$ & $\begin{array}{c}10 \\
(76.9)\end{array}$ \\
\hline & Beef at abattoir & 3 & 0 & 0 & 0 & $\begin{array}{c}2 \\
(66.7)\end{array}$ & $\begin{array}{c}2 \\
(66.7)\end{array}$ & $\begin{array}{c}3 \\
(100)\end{array}$ & $\begin{array}{c}2 \\
(66.7)\end{array}$ \\
\hline & Beef at butcher & 7 & 0 & 0 & 0 & $\begin{array}{c}1 \\
(14.2)\end{array}$ & $\begin{array}{c}3 \\
(42.9)\end{array}$ & $\begin{array}{c}4 \\
(57.1)\end{array}$ & $\begin{array}{c}2 \\
(28.6)\end{array}$ \\
\hline & Total & 23 & $2(8.7)$ & $\begin{array}{c}3 \\
(13.1)\end{array}$ & $\begin{array}{c}3 \\
(13.0)\end{array}$ & $\begin{array}{c}10 \\
(43.5)\end{array}$ & $\begin{array}{c}13 \\
(56.5)\end{array}$ & $\begin{array}{c}17 \\
(73.9)\end{array}$ & $\begin{array}{c}14 \\
(60.9)\end{array}$ \\
\hline \multirow[t]{4}{*}{ CNS } & Environment ${ }^{* *}$ & 21 & 0 & $\begin{array}{c}1 \\
(4.8)\end{array}$ & 0 & 0 & $\begin{array}{c}3 \\
(14.3)\end{array}$ & $\begin{array}{c}1 \\
(4.8)\end{array}$ & $\begin{array}{c}2 \\
(9.5)\end{array}$ \\
\hline & Beef at abattoir & 5 & 0 & 0 & $\begin{array}{c}1 \\
(20.0)\end{array}$ & $\begin{array}{c}1 \\
(20.0)\end{array}$ & 0 & $\begin{array}{c}2 \\
(40.0)\end{array}$ & $\begin{array}{c}1 \\
(20.0)\end{array}$ \\
\hline & Beef at butcher & 11 & $\begin{array}{c}1 \\
(9.1)\end{array}$ & 0 & $\begin{array}{c}1 \\
(9.1)\end{array}$ & $\begin{array}{c}1 \\
(9.1)\end{array}$ & $\begin{array}{c}3 \\
(27.3)\end{array}$ & 0 & $\begin{array}{c}4 \\
(36.4) 4\end{array}$ \\
\hline & Total & 37 & $\begin{array}{c}1 \\
(2.7)\end{array}$ & $\begin{array}{c}1( \\
2.7)\end{array}$ & $\begin{array}{c}2 \\
(5.4)\end{array}$ & $\begin{array}{c}2 \\
(5.4)\end{array}$ & $\begin{array}{c}6 \\
(16.2)\end{array}$ & $\begin{array}{c}3 \\
(8.1)\end{array}$ & $\begin{array}{c}7 \\
(18.9)\end{array}$ \\
\hline
\end{tabular}

Note: $\mathrm{C}=$ Chloramphenicol $; \mathrm{CN}=$ Gentamycin $; \mathrm{OT}=$ Oxytetracycline $; \mathrm{OX}=$ Oxacillin; $\mathrm{PB}=$ Polymyxin B; $\mathrm{SXT}$

= Trimethoprim-Sulfamethoxazole; $\mathrm{W}=$ Trimethoprim; $*$ = TE resistant isolates were not observed; ${ }^{* *}=$ Abat-

toir Environment*

\section{Resistance pattern}

Out of the tested 60 isolates, $31(51.7 \%)$ were resistant to one drug to multiple of six drugs (Table 8). Ten isolates for single drug, four for two drugs but the remaining 17 for multiple of three to six drugs resistance (MDR) were observed. Higher isolates of 5 were found resistant to four dug combination of oxytetracycline-polymyxin B-trimethoprim-sulfamethoxazole-trimethoprim (OT-PB-SXT-W). 
Table 8: Single to multiple of six drug resistant patterns of the isolates at Addis Ababa Abattoir Enterprise beef abattoir line.

\begin{tabular}{|c|c|c|c|c|}
\hline $\begin{array}{l}\text { Resistant isolates } \\
\text { pattern }\end{array}$ & Drugs of resistant & $\begin{array}{l}\text { No. of } S . \\
\text { aureus }\end{array}$ & $\begin{array}{l}\text { No. of } \\
\text { CNS }\end{array}$ & No. of Total \\
\hline \multirow[t]{6}{*}{ Single drug resistant } & $\mathrm{C}$ & 0 & 1 & 1 \\
\hline & $\mathrm{OT}$ & 0 & 3 & 3 \\
\hline & OX & 0 & 1 & 1 \\
\hline & PB & 2 & 1 & 3 \\
\hline & W & 0 & 2 & 2 \\
\hline & Subtotal № (\%) & $2(11.1)$ & $8(61.5)$ & $10(32.3)$ \\
\hline \multirow[t]{4}{*}{ Two drugs resistant } & $\mathrm{C}-\mathrm{W}$ & 0 & 1 & 1 \\
\hline & OT-PB & 2 & 0 & 2 \\
\hline & PB-W & 1 & 0 & 1 \\
\hline & Subtotal № (\%) & $3(16.7)$ & $1(7.7)$ & $4(12.9)$ \\
\hline \multirow[t]{5}{*}{ Three drugs resistant } & C-OT-W & 0 & 1 & 1 \\
\hline & OT-SXT-W & 0 & 1 & 1 \\
\hline & OT-PB-W & 2 & 1 & 3 \\
\hline & PB-SXT-W & 1 & 1 & 2 \\
\hline & Subtotal № (\%) & $3(16.7)$ & $4(30.8)$ & $7(22.6)$ \\
\hline \multirow[t]{3}{*}{ Four drugs resistant } & OT-PB-SXT-W & 5 & 0 & 5 \\
\hline & OX-PB-SXT-W & 1 & 0 & 1 \\
\hline & Subtotal №. (\%) & $6(33.3)$ & 0 & $6(19.4)$ \\
\hline \multirow[t]{4}{*}{ Five drugs resistant } & C-CN-OT-SXT-W & 1 & 0 & 1 \\
\hline & OT-OX-PB-SXT-W & 1 & 0 & 1 \\
\hline & C-OT-PB-SXT-W & 1 & 0 & 1 \\
\hline & Subtotal № (\%) & $3(16.7)$ & 0 & $3(9.7)$ \\
\hline Six drugs resistant & C-CN-OT-OX-PB-W & $1(5.6)$ & 0 & $1(3.2)$ \\
\hline Total № (\%) & 31 & $18(100)$ & $13(100)$ & $31(100)$ \\
\hline
\end{tabular}

\section{Discussion}

\section{Prevalence of Staphylococcus}

Meat is an important vehicle for the transfer of antibiotic resistances from animals to humans, and antimicrobial resistance has always been a major concern for nosocomial infections in hospital environments. Such transfer can occur in three ways: by means of antibiotic residues in food, through the transfer of resistant food borne pathogens, or through the ingestion of resistant parts 
of the original food microflora and resistance transfer to pathogenic microorganisms (Kruse and Sorum, 1994; Klein, 1999; Teuber, 1999; Mayrhofer et al., 2004). The present overall prevalent of $35.5 \%$ of Staphylococcus is slightly lower than the $42.8 \%$ total Staphylococcal reported by Gizaw (2014). However, there is similarity in prevalence of $S$. aureus at hooks (18.2\%) and personnel hand swab (46.2\%). The raising Staphylococcal load from $23.5 \%$ at ready for distribution at abattoir to $52.9 \%$ butchers house indicated the high risk of beef contamination by $S$. aureus carriers human being and from the beef handling environment like transporting trucks (45.5\%) along the meat value chain (Gudeta, 2012). Asymptomatic nasal carriers of S. aureus (Kluytmans et al., 1997; Gorwitz et al., 2008; Gizaw, 2014) as a normal flora with human an important source of spread of $S$. aureus to food and food production and processing chain were also indicated by Miller and Diep (2008), Kazakova et al. (2005) and Muto et al. (2003). This was supported by $46.2 \%$ Staphylococcal isolation on personnel's hands swabs observed from the current study.

The present findings of $S$. aureus (8.8\%) and CNS (14.7\%) were lower than the $S$. aureus prevalence $38.5 \%$ from carcass swab (Gizaw, 2014), 28.57\% from poultry and $29.41 \%$ from beef, $23.86 \%$ S. aureus from raw meat (Pesavento et al., 2007) but similar was $15.15 \%$ from pork (Pesavento et al., 2007). But the prevalence on ready for distribution and the $20.6 \%$ S. aureus and $32.4 \%$ CNS were similar with reports of Gizaw (2014). This variation could due to difference in study area and sources of sample. Moreover, the present concomitant raising of $S$. aureus $(8.8 \%)$ and $\mathrm{CNS}(14.7 \%)$ at ready for distribution to raise to $20.6 \%$ and $32.4 \%$ respectively at post-delivery at public supply could be due to further contamination of the beef product at postharvest stages followed by growth of microbial. These indicate the increasing in rate of contamination and multiplication of Staphylococcal on the carcasses and along the beef handling stage. Rani et al. (2017) suggested meat distribution stage as the most critical period where the quality can easily be compromised. During distribution, pathogenic and spoilage micro-organisms may grow (Rani et al., 2017).

\section{DNase and Coagulase test}

Coagulase testing was used as gold standard for differentia between S. aureus and coagulase negative Staphylococcus (CNS) (Winn et al., 2006). Clinical S. aureus isolation requires a cost effective and better tests in resource limited developing countries (Kateete et al., 2010) where tube plasma coagulase test is confirmatory. However, as an alternative diagnostic tool, DNase test can assist 
or replace it. The present finding showed almost perfect agreement $($ Kappa $=$ $0.86,95 \% \mathrm{CI}=0.73-0.99$ ) between the DNase and tube plasma coagulase tests in screening S. aureus from the CNS. According to Viera and Garrett (2005), almost perfect test agreement ranges from 0.81-0.99.

The present $91.3 \%$ sensitivity was found in agreement and lower than 94-100\% previous findings reported by other investigators (Tager et al., 1948, Orth et al., 1971; Oranusi and Umoh, 2006) but higher than the $75-76 \%$ sensitivity reported by Kateete et al. (2010) using tri-combination test (human or sheep plasma/Mannitol salt agar/DNase). The differences could be due to difference in the test method where by Kanep agar and rabbit plasma were used in this study. The present $94.6 \%$ specificity is found lower than the $100 \%$ reported by Kateete et al., (2010) whose used tri-combination test both by (human plasma/Mannitol salt agar/DNase). "The two DNase negative S. aureus" in the context of tube coagulases the gold standard, DNase negative but tube coagulases positive are false negatives isolates. They may probably be MRSA isolates which are reported to react weakly. Presence of such rare coagulase negative S. aureus strains has been reported by Koneman et al. (1997) which could weakly coagulase reacting strain termed as MRSA or Staphylococcus schleiferi subsp coagulans. (Kateete et al., 2010). Again, Rao et al. (2002) also reported DNase negative $\mathbf{S}$. aureus. On the other hand, the present two isolates with DNase positive but tube coagulase negative (i.e. false positive isolates) were presume Staphylococcus schleiferi subsp. coagulans. Kateete et al. (2010) also observed isolates with such property with similar assumption in Uganda.

\section{Drug resistance profile of Staphylococcal species}

The rapidly increasing in the number of antimicrobial resistant bacteria agents could be due to the extended use and misuse of antibiotics in livestock farming and human diseases treatment). Staphylococcal species (Kitai et al., 2005; Becker et al., 2014), have shown a considerable increase in resistance against most antibiotics (Valsangiacomo et al., 2000) with the consequence of prolonged hospital stay and increased costs of treatment (Kitara et al., 2011).

Tetracycline resistant isolate was not observed in this study but $62.5 \%$ were reported from milk (Gizaw, 2014). Again, unlike the present finding, 19.0\% S. aureus were resistant to tetracycline with $8.3 \%, 20.0 \%$ and $25.0 \%$ from poultry, beef and pork were reported (Pesavento et al., 2007). This could be due to the difference among the sources of isolates and geographical location. On 
the other hand, the $31.7 \%$ resistant isolate to oxytetracycline observed in this study could be from frequently use of this in Ethiopia in animal's treatment (DACA, 2009) which might develop drug resistance. Present finding showed higher resistant $S$. aureus (43.5\%) than CNS (5.4\%) to co-trimoxazole indicating greater risk of resistance development of the pathogenic strain than the less pathogenic group to commonly used drug. The current $20.0 \%$ resistance to co-trimoxazole was lower than the resistant $S$. aureus from outpatients (53.6\%) and inpatients (44.7\%) (Kitara et al., 2011). The overall 6.7\% oxacillin resistant Staphylococci with S. aureus (13.1\%) and CNS (2.7\%) were lower than the $35.7 \%$ resistant $S$. aureus isolates (Pesavento et al., 2007). This finding is still lower than the $66.7 \%$ from poultry and $30.0 \%$ from pork oxacillin resistant $S$. aureus. But the present oxacillin resistant $S$. aureus was similar with the $10.0 \%$ reports from beef meat items (Pesavento et al., 2007). The current over all $5.0 \%$ resistance to gentamycin was similar with a pooled $9.5 \%$ resistant $S$. aureus reported by Pesavento et al. (2007) but lower than the $16.7 \%, 10.0 \%$ and $5.0 \%$ from poultry, beef and pork (Pesavento et al. (2007) respectively.

The present $15.4 \%$ to $76.9 \%$ resistant $S$. aureus to chloramphenicol, gentamycin, oxytetracycline, oxacillin, polymyxin B, trimethoprim-sulfamethoxazole and trimethoprim were observed at abattoir environment could be due to contamination of the abattoir environment from animals, or from carrier humans particularly from abattoir workers harboring resistant strain. Evidence that transfer of antimicrobial resistance from food-producing animals to humans directly via the food chain as route of spread were documented (Wooldridge, 2012). The absence of resistant Staphylococcus isolates to chloramphenicol, gentamycin and oxacillin on beef both at abattoir and butchery could be due to the fact that these drugs are not used in animal treatment in Ethiopia where no development of resistant strain. The occurrence of one or more resistant almost along the studied beef line location indicates the contamination of environmental sites which concerned as one routes of resistant isolate circulation via contact. Contaminated environmental sites were suggested as pathway for the transfer of resistant isolate in meat (Wooldridge, 2012; Bengtsson-Palme et al., 2018). Moreover, transfer of resistant genetic elements between bacteria in mixed populations as a cross resistance to drugs of similar generic groups could make complex potential routes of resistant isolate spread. Similar suggestions were given (Wooldridge, 2012; Bengtsson-Palme et al., 2018).

Total of $51.7 \%$ resistant isolates to one drug to multiple of six drugs consisting as multiple drugs resistance (MDR) were observed. A large proportion 
(89.3\%) from food line (Gizaw, 2014), 53.7\% from hospital cases (Gizachew et al., 2015) were showed MDR in Ethiopia. The presences of nasal carriage of resistance staphylococcus were also reported by Tewodros and Gedebou (1984) in Ethiopia. Like the present 51.7\% MDR isolation, Pesavento et al. (2007) also reported $30.95 \%$ resistant isolates to at least three antibiotics in indicating significance resistance among these pathogens.

\section{Conclusion and recommendation}

The present finding indicates increased contamination of meat product along handling line with both by $S$. aureus and CNS with more than half percent of the isolates being resistant to single to multiple drug resistant. Thus, this finding warrants the application of good hygienic practices along the beef abattoir line with control of drug resistance. The DNase test showed strong agreement with tube coagulase test with low false positive and negative isolates indicated the possible alternative use of these diagnostic tests for screening staphylococcal isolates in resource-limited settings like Ethiopia.

\section{Acknowledgement}

Cooperation of Addis Ababa Abattoir Enterprise staff in providing the requested sample on time was highly appreciated. Prof. Fries Reinhard was highly appreciated for microbiologic media support and Prof. Gobena Ameni provided laboratory equipment. Aklilu Lemma Institute of Pathobiology Addis Ababa University was appreciated for provision of laboratory space.

\section{Conflict of interest}

There is no conflict of interest to declare

\section{References}

Bauer, A.W., Kirby, W.M.M., Sherris, J.C., Turck. M., 1966. Antibiotic susceptibility testing by standard single disc method. Am. J. Clin. Patholo., 45(4), 493-496.

Becker, K., Heilmann, C., Peters, G., 2014. Coagulase-Negative Staphylococci, Clin. Microbiol. Rev., 27 (4), 870-926. doi:10.1128/CMR.00109-13.

Bengtsson-Palme, J., Kristiansson, E., Larsson, D.G.J., 2018. Environmental factors influencing the development and spread of antibiotic resistance. FEMS Microbiol. Rev., 42(1), fux053. doi: 10.1093/femsre/fux053. 
CLSI, 2012. Clinical and Laboratory Standards institute (CLSI). "Performance standard for antimicrobial disk and dilution susceptibility tests for Bacterial isolation from animal.

DACA, 2009. Antimicrobial use, resistance and containment baseline survey syntheses of findings. Drug Administration and Control Authority (DACA), 2009, Addis Ababa, Ethiopia.

Eshetie, S., Tarekegn, T., Moges, F., Amsalu, A., Birhan, W., Huruy. K., 2016. Methicillin resistant Staphylococcus aureus in Ethiopia: a meta-analysis. BMC. Infect. Dis., 16, 689. doi 10.1186/s12879-016-2014-0.

Gizachew, M., Abdella, H., Tiruneh, M., 2015. Antimicrobial Susceptibility Patterns of Staphylococcus aureus at the University of Gondar Tertiary Hospital, Northwest Ethiopia: A Retrospective Cross Sectional Study. J. Bacteriol. Parasitol., 6 (3), 228. doi:10.4172/2155-9597.1000228.

Gizaw, F., 2014. Staphylococcus: Epidemiology and its drug resistance in cattle, food Chains and humans in central Ethiopia, MSc thesis. Addis Ababa University, College of Veterinary Medicine and Agriculture, Bishoftu, Ethiopia.

Gorwitz, R.J., Kruszon-Moran, D., McAllister, S.K., McQuillan, G., McDougal, L.K., Fosheim, G.E., Jensen, B.J., Killgore, G., Tenover, F.C., Kuehnert, M.J., 2008. Changes in the prevalence of nasal colonization with Staphylococcus aureus in the United States 2001-2004. J. Infect. Dis., 197(9), 1226-1234. doi: 10.1086/533494.

Gudeta, B., 2012. Optimizing logistic chain of animal transport and meat distribution: studies on livestock markets and abattoirs in Addis Ababa City. MSc thesis, Addis Ababa University, Civil Engineering/Road and Transport Engineering Stream, Addis Ababa, Ethiopia.

Kateete, D.P., Kimani, C.N., Katabazi, F.A., Okeng, A., Okee, M.S., Nanteza, A., Joloba, M.L., Najjuka, F.C., 2010. Identification of Staphylococcus aureus: DNase and Mannitol salt agar improve the efficiency of the tube coagulase test. Ann. Clin. Microbiol. Antimicrob., 9, 23. doi: 10.1186/1476-0711-9-23.

Kazakova, S.V., Hageman, J.C., Matava, M., Srinivasan, A., Phelan, L., Garfinkel, B., Boo, T., McAllister, S, Anderson, J., Jensen, B., Dodson, D., Lonsway, D., McDougal, L.K., Arduino, M., Fraser, V.J., Killgore, G., Tenover, F.C., Cody, S., Jernigan, D.,B., 2005. A clone of methicillin-resistant Staphylococcus aureus among professional football players. N. Engl. J. Med., 352(5), 468-475. doi: 10.1056/NEJMoa042859.

Kluytmans, J., van Belkum, A., Verbrugh, H., 1997. Nasal carriage of Staphylococcus aureus: epidemiology, underlying mechanisms, and associated risks. Clin Microbiol. Rev., 10(3), 505-520.

Ethiop. Vet. J., 2019, 23 (1), 90-110 
Koneman, E.W.A.S., Janda, W.M., Schreckenberger, P.C., Winn, W.C., 1997. The Gram-positive cocci: Staphylococci and related organims. In Color Atlas and Textbook of Diagnostic Microbiology. Philadelphia: Lippincott-Raven Koneman EW, 5, 551-576.

Lowry, F.D., 1998. Staphylococcus aureus infections. New. Engl. J. Med., 339, 520-532. doi: 10.1056/NEJM199808203390806.

Lowy, F.D., 2003. Antimicrobial resistance: the example of Staphylococcus aureus. J. Clin. Invest., 111(9), 1265-1273. doi: 10.1172/JCI200318535.

Miller, L.G., Diep, B.A., 2008. Clinical practice: colonization, fomites, and virulence: rethinking the pathogenesis of community-associated methicillin-resistant Staphylococcus aureus infection. Clin. Infect. Dis., 46, 752-760. doi: 10.1086/526773.

Monttville, T.J., Matthews, K.R., Kniel, K.E., 2012. Food microbiology: An introduction, $3^{\text {rd }}$ edition. Washington, ASM Press $570 \mathrm{p}$.

Muto, C.A., Jernigan, A.J., Ostrowsky, B.E., Richet, H.M., Jarvis, W.R., Boyce, J.M., Farr, B.M., 2003. SHEA guideline for preventing nosocomial transmission of multidrug-resistant strains of Staphylococcus aureus and enterococcus. Infect. Control Hosp. Epidemiol., 24(5), 362-86. doi: 10.1086/502213.

Oranusi, S.G, Umoh, V.J, 2006. Toxicity test and bacteriophage typing of Staphylococcus aureus isolates from food contact surfaces and foods prepared by families in Zaria, Nigeria. Afr. J. Biotech., 5(4):362-365.

Orth, D.S., Chugg, L.R., Anderson, A.W., 1971. Comparison of Animal Sera for Suitability in Coagulase Testing. Appl. Environ. Microbiol., 21(3), 420-425. PMC: 377196.

Pesavento, G., Ducci, B., Comodo, N., Nostro, A., 2007. Antimicrobial resistance profile of Staphylococcus aureus isolated from raw meat: A research for methicillin resistant Staphylococcus aureus (MRSA). Food Control, 18(3), 196-200. doi. org/10.1016/j.foodcont.2005.09.013

Rani, Z. T., Hugo, A., Hugo, C. J., Vimiso, P., Muchenje, V., 2017. Effect of post-slaughter handling during distribution on microbiological quality and safety of meat in the formal and informal sectors of South Africa: A review. S. Afr. J. Anim. Sci., 47 (3), 255-267.

Rao, J.G., Qamruddin, A.O., Hassan, I.A., Burnie, J.P., Ganner, M., 2002. Cluster of clinical isolates of epidemic methicillin-resistant Staphylococcus aureus (EMRSA) with a negative deoxyribonuclease (DNase) test-implications for laboratory diagnosis and infection control. J. Hosp. Infect., 51(3), 238-239.

Tager, M., Hales, H.B., 1948. The Experimental Production of Antibodies to Staphylocoagulase. J Immunol., 60 (4), 475-485. PMID: 18106198 
Tewodros, W., Gedebou, M., 1984. Nasal carrier rates and antibiotic resistance of Staphylococcus aureus isolates from hospital and non-hospital populations, Addis Ababa. Trans. R. Soc. Trop. Med. Hyg., 78 (3), 314-318. PMID: 6464129.

USDA, 2012. Introduction to the microbiology of food processing. Small plant news guidebook series United States Department of Agriculture (USDA) Food Safety and Inspection Service. August 2012, pp. 1-64.

Viera, A.V., Garrett, J.M., 2005. Understanding Interobserver Agreement: The Kappa Statistic, Fam. Med., 37(5), 360-363.

Waldvogel, F.A., 1995. Staphylococcus aureus (including toxic shock syndrome). In: Mandell, G.L., Bennett, J.E., Dolin, R., editors. Mandell, Douglas and Bennett's Principles and Practice of Infectious Diseases, $4^{\text {th }}$ Edn. New York, Churchill Livingstone, pp. $1754-1777$.

Winn, W., Allen, S., Janda, W., Koneman, E., Procop, G., Schreckenberger, P., Woods, G., 2006. Koneman's Color Atlas and Textbook of Diagnostic Microbiology. 6th Edition, Lippincott Williams and Wilkins, New York

Wooldridge, M. 2012. Evidence for the circulation of antimicrobial-resistant strains and genes in nature and especially between humans and animals. Rev.Sci. Tech., 31(1), 231-247.

Ethiop. Vet. J., 2019, 23 (1), 90-110 